\title{
Conductance recovery and spin polarization in boron and nitrogen co-doped graphene nanoribbons
}

\author{
Seong Sik Kim, ${ }^{a}$ Han Seul Kim, ${ }^{a}$ Hyo Seok Kim, ${ }^{a}$ and Yong-Hoon Kim ${ }^{a, b}$ * $^{*}$ \\ ${ }^{a}$ Graduate School of EEWS, KAIST, 291 Daehak-ro, Yuseong-gu, Daejeon 305-701, Korea. \\ ${ }^{\mathrm{b}}$ KI for the NanoCentury, KAIST, 291 Daehak-ro, Yuseong-gu, Daejeon 305-701, Korea.
}

Abstract

We present an ab initio study of the structural, electronic, and quantum transport properties of B-N-complex edge-doped graphene nanoribbons (GNRs). We find that the B-N edge codoping is energetically a very favorable process and furthermore can achieve novel doping effects that are absent for the single $\mathrm{B}$ or $\mathrm{N}$ doping. The compensation effect between $\mathrm{B}$ and $\mathrm{N}$ is predicted to generally recover the excellent electronic transport properties of pristine GNRs. For the zigzag GNRs, however, the spatially localized B-N defect states selectively destroy the doped-side spin-polarized GNR edge currents at the valence and conduction band edges. We show that the energetically and spatially spin-polarized currents survive even in the fully ferromagnetic metallic state and heterojunction configurations. This suggests a simple yet efficient scheme to achieve effectively smooth GNR edges and graphene-based spintronic devices.

* Corresponding author. Tel.: +82 42350 1717. E-mail address: y.h.kim@kaist.ac.kr (Y.-H. Kim) 


\section{Introduction}

Adopting the nanoribbon form of graphene [1] is an attractive route toward the nextgeneration graphene-based electronic [2-5], optoelectronic [6], and spintronic applications [7-11], thanks to the tunable quantum-confined bandgaps [12-15] and spin-polarized edge states [7, 15-18]. For example, room-temperature ballistic charge transport on a length scale greater than ten micrometers was recently demonstrated [19]. To extract such exceptional properties, narrow and clean graphene nanoribbons (GNRs) with atomically smooth edges are required $[2,3,15,19-21]$. On the other hand, to tune their electronic and charge transport properties for practical device applications, one usually needs to functionalize the GNRs by introducing defects and dopants [22-24]. The challenge is thus to devise a method to controllably functionalize GNRs while maintaining their desirable and unique electronic and transport properties.

In this article, carrying out density functional theory (DFT) and DFT-based nonequilibrium Green's function (NEGF) calculations, we study the structural, electronic, and charge transport properties of B-N-complex edge-doped armchair and zigzag GNRs (aGNRs and zGNRs, respectively). Boron and nitrogen are ideal doping candidates for graphene and GNRs, and significant efforts have been devoted toward the understanding of their physical properties and realization of practical devices based on them [22, 25-31]. However, while theoretical studies have been carried out on single B/N-doped [25-31] or separate B-N codoped GNRs [30], introducing B-N-complex units into GNRs still remains unexplored. We will show that binary B-N-complex GNR edge doping is energetically a very favorable process, and moreover it almost perfectly recovers the excellent charge transport property of pristine GNRs. For the zGNRs, in particular, we will demonstrate that it can support spatially and energetically spin-polarized currents even without magnetic electrodes and/or external 
fields. Two heterojunction examples will be provided where the zGNRs are utilized as the spin current channel and spin injection electrodes, respectively.

\section{Computational method}

We considered both the armchair and zigzag GNRs labeled by the number $N$ of respectively zigzag chains $(N$-aGNRs) and dimer lines $(N$-zGNR) contained in their unit cells. Starting from the pristine GNRs, we introduced single B, N, and B-N-complex dopant atoms into the nanoribbon edges, which were found to be the most favorable doping sites [27, 31] (see Supplemental Data Table S1). In all the cases, pristine or doped GNR edges were terminated by hydrogen atoms. Rectangular simulation boxes with periodic boundary condition were employed, and the inter-GNR distance was kept to a minimum of $20.0 \AA$ to avoid interactions with images.

We carried out DFT calculations within the local density approximations [32] using SIESTA software [33]. The atomic cores were replaced by norm-conserving nonlocal pseudopotentials of Troullier-Martins type [34]. The double $\zeta$-plus-polarization-level numerical atomic orbital basis sets whose extensions were defined by the $80 \mathrm{meV}$ energy cut-off were adopted. The real-space integration was done on the mesh defined by 200 Ry kinetic energy cutoff. The transmission functions of single $\mathrm{B}, \mathrm{N}$ and $\mathrm{B}-\mathrm{N}$-complex doped GNRs were calculated using the fully self-consistent DFT-based NEGF method, as implemented in the TranSIESTA code [35]. The surface Green's functions were extracted from two independent DFT calculations for the two (four) pristine aGNR (zGNR) unit cells that correspond to the electrodes with a $96 \overrightarrow{k_{\perp}}$-point sampling along the charge transport direction (see Supplemental Data Fig. S1). In calculating the transmission functions $T(E)$, the energy window was scanned from -1.5 to $1.5 \mathrm{eV}$ near the Fermi level $E_{F}$ with the $0.001 \mathrm{eV}$ step. In many cases, using our inhouse code $[36,37]$ and/or the generalized gradient approximation, we have crosschecked the 
validity of our results (see Supplemental Data Fig. S2). We emphasize that the edge N doping has been experimentally achieved already [21].

\section{Results and Discussion}

We start with discussing the energetics of B-N-complex doping. Figure 1 summarizes the formation energies of the six-unit-cell-long (length $L=25.56 \AA$ ) 11 -aGNRs (width $W=14.21$ $\AA)$ and 12-unit-cell-long $(L=29.52 \AA) 6$-zGNRs $(W=13.58 \AA)$ doped by binary B and N atoms at one GNR edge in various relative configurations, compared with those of the energetically most stable single-N and single-B doping configurations (edge and next-edge sites, respectively. See also Supplemental Data Fig. S1 and Table S1 for other doping configurations including the GNR interior sites). The formation energies of the B-N doped GNRs were computed using the following equation [28]:

$$
E_{f}=E_{\text {doped }}-E_{\text {pristine }}+2 \mu_{C}-\mu_{B}-\mu_{N}
$$

where $E_{\text {doped }}$ and $E_{\text {pristine }}$ are the total energies of the doped/pristine GNRs, and $\mu_{C / B / N}$ are the chemical potential energies of an atomic species $\mathrm{C} / \mathrm{B} / \mathrm{N}$, respectively. The chemical potentials of $\mathrm{C}, \mathrm{B}$, and $\mathrm{N}$ were extracted from graphene, $\alpha$-phase bulk $\mathrm{B}$, and $\mathrm{N}_{2}$ gas, respectively. For both aGNR and zGNR cases, we found that the energetically most stable atomic configurations are those where the $\mathrm{B}$ and $\mathrm{N}$ are chemically bonded to each other and form a complex at the GNR edges. In particular, we note that B-N-complex edge doping is energetically even more favorable than single B or N doping in both aGNRs and zGNRs. 
(a) 11-aGNR
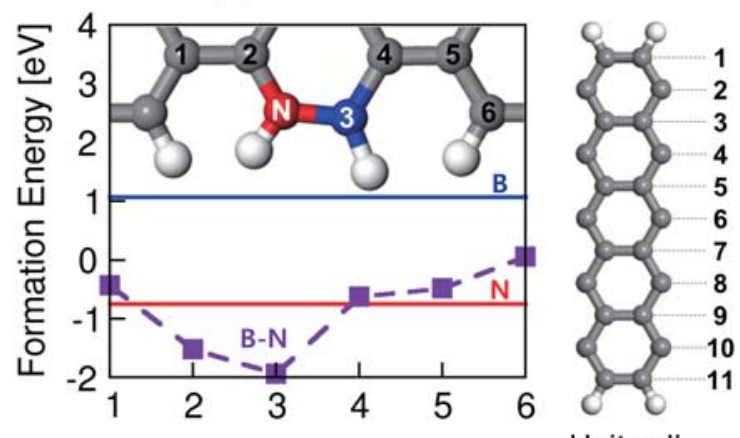

(b) 6-zGNR
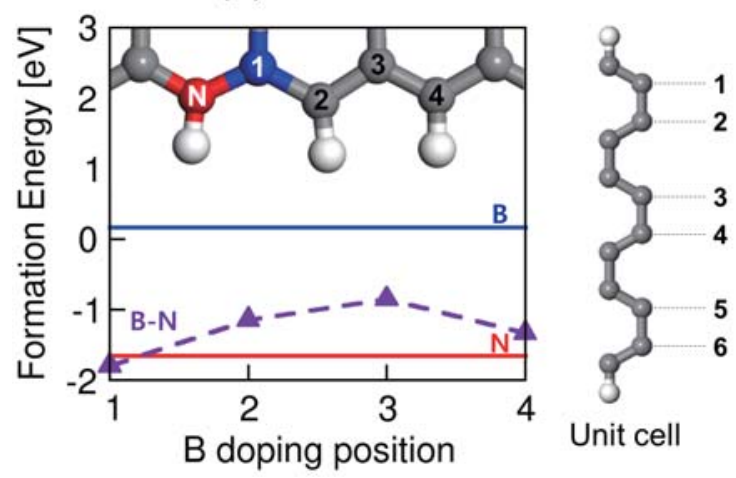

Fig. 1 Formation energies of single B-, single N- and binary B-N-doped (a) 11-aGNRs and (b) 6-zGNRs. Insets: Doping sites correspond to the B doping positions. The atomic models on the right-hand side represent the unit cell of the 11-aGNR and 6-zGNR respectively.

We now consider the electronic and charge transport properties of the energetically most favorable B-N-complex edge-doped aGNRs. As references, we first show in Figs. $2 \mathrm{a}$ and $2 \mathrm{~b}$ (see also Supplemental Data Fig. S3) the transmissions of single B and single N edge-doped 11-aGNRs, respectively. The data obtained for the pristine 11-aGNR are also shown together as dotted lines. In agreement with previous studies [29, 31], we observe that transmissions are significantly reduced from the pristine GNR values of $1 G_{0}\left(=e^{2} / h\right)$ in the valence/conduction band regions (p-/n-types) for the single B/N-doped 11-aGNRs (Figs. 2a/2b). Namely, the single $\mathrm{B} / \mathrm{N}$ dopant atom behaves as an acceptor/donor impurity in aGNRs, as can be directly visualized in the energy-resolved local density of states (LDOS) plots for the valence band (shaded area in Fig. 2a)/conduction band energy regions (shaded area in Fig. 2b) shown in 
Figs. 2d/2c.

(a) B doped

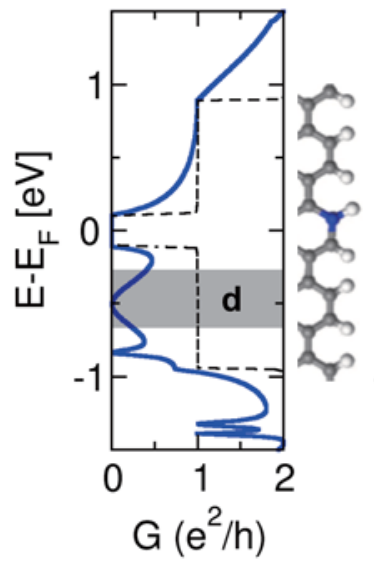

(e) B-N doped

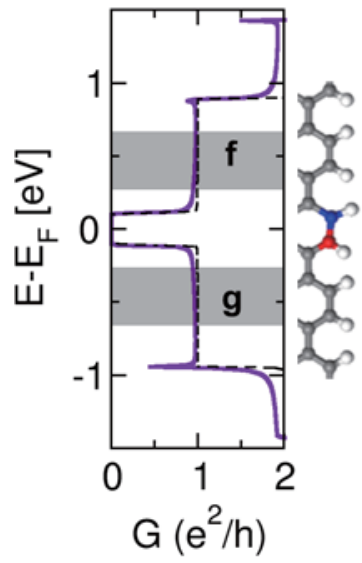

(b) $\mathrm{N}$ doped

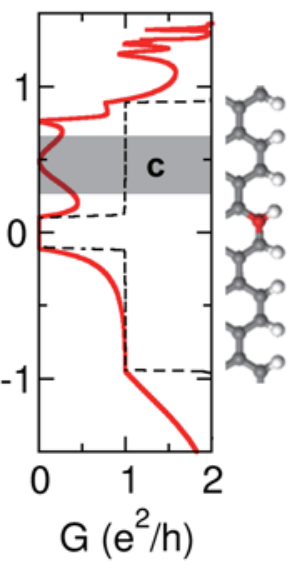

(f)

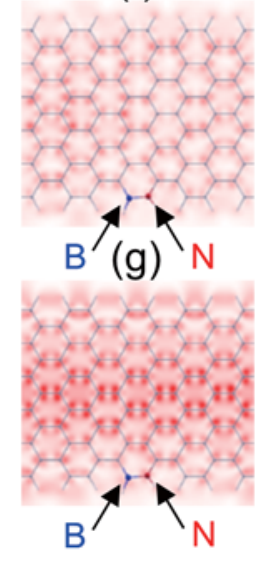

(c)

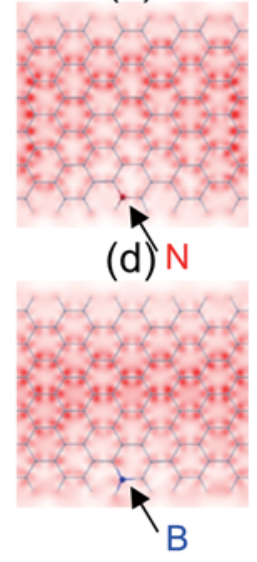

(h)

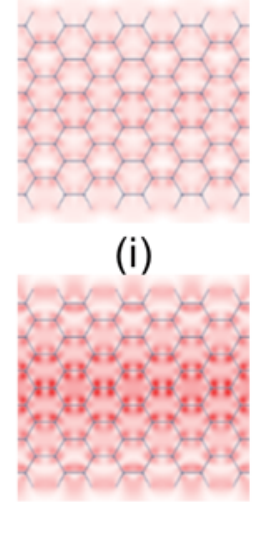

0.03

O

Fig. 2 Transmissions of (a) single B-doped, (b) single N-doped, (e) B-N-complex doped 11aGNRs. Transmissions of the pristine 11-aGNRs are shown together (dotted lines). LDOS of the (c) single N-doped, (d) single B-doped, (f,g) B-N-complex doped, and (h,i) pristine 11aGNRs for the energy ranges $E_{F}+0.3 \mathrm{eV} \sim E_{F}+0.7 \mathrm{eV}((\mathrm{c}),(\mathrm{f})$ and $(\mathrm{h}))$ and $E_{F}-0.7 \mathrm{eV} \sim$ $E_{F}-0.3 \mathrm{eV}((\mathrm{d}),(\mathrm{g})$ and $(\mathrm{i}))$.

Compared with the single $\mathrm{B} / \mathrm{N}$ doping cases, we find that $\mathrm{B}-\mathrm{N}-$ complex edge doping almost perfectly recovers the transmission of pristine aGNRs (Fig. 2e) (see also Supplemental Data Fig. S3). This can be easily understood based on the chemical valency of the connected 
$\mathrm{B}$ and $\mathrm{N}$ atoms (three and five, respectively), which should recover that of the $s p^{2} \mathrm{C}$ network due to the compensation effect. The conductance recovery is again well demonstrated in the energy-resolved LDOS plots shown in Figs. $2 \mathrm{f}$ and $2 \mathrm{~g}$, which almost match those of a pristine GNR shown in Figs. $2 \mathrm{~h}$ and $2 \mathrm{i}$. Note that experimentally preparing pristine GNRs with atomically smooth edges is still a formidable challenge $[2,15,20,21]$. In this context, we propose the B-N-complex doping as a practical solution to anneal the edge defects and accordingly realize "effectively" clean and smooth GNR edges. Combined with the energetic consideration presented above, this result should have significant practical implications, e.g, in the lithographic patterning of GNR devices.

We next move on to the case of zGNRs. In Figs. 3a and 3b (See also Supplementary data Fig. S4), as references, we show the transmissions obtained from the single $\mathrm{B}$ and single $\mathrm{N}$ edge doped 6-zGNRs, respectively. In the ground state of pristine zGNRs, the spins along each zigzag edge are ferromagnetically aligned whereas two edge states are antiferromagnetically (AF) ordered. This ground state presents the degenerate $\alpha$ - and $\beta$-spin bands with a bandgap inversely proportional to the ribbon width and the resulting gapped spin-degenerate transmission spectrum (shown as dotted lines in Figs. 3a and 3b) with zero total spin polarization [7]. The spatial and energetic distributions of these spin states can be visualized in the net-spin $(\alpha-\beta)$ LDOS plots (Figs. 3d and 3e), which show that the edgeoriginated spin states change sign as one moves from the valence to conduction bands.

Upon introducing single substitutional $\mathrm{B}$ and $\mathrm{N}$ dopant atoms into the zGNR edge, in agreement with previous studies [25-31], we obtain the n-type (Fig. 3a) and p-type (Fig. 3b) transport polarities, respectively. This anomalous acceptor-donor transition results from the Coulomb repulsion between the localized zigzag edge states and the dopant atom states [2931]. The quasi-bound states induced by a $\mathrm{B}$ or $\mathrm{N}$ atom break the spin degeneracy, but the the robustness and accessibility of spin polarization is limited in that broad and deep conductance 
drops appear in both spin channels. This results from the spatially rather long-ranged nature of the defect states, which destroy even the spin currents along the opposite undoped GNR edges (Figs. 3f and 3g).

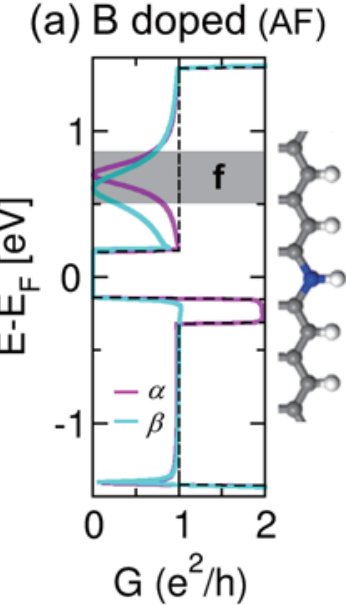

(d)

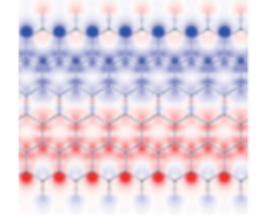

(e)

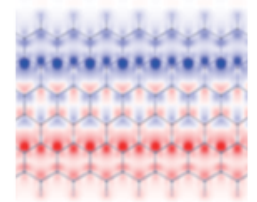

(b) $\mathrm{N}$ doped (AF)

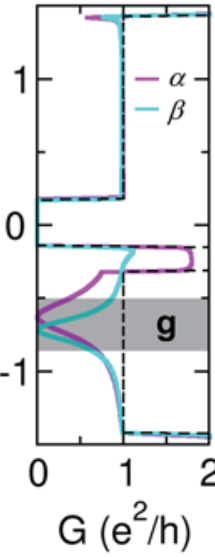

(f)

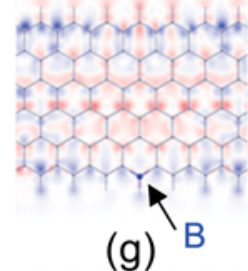

(g)

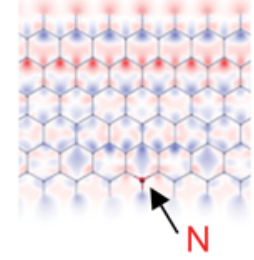

(c) B-N doped (AF)

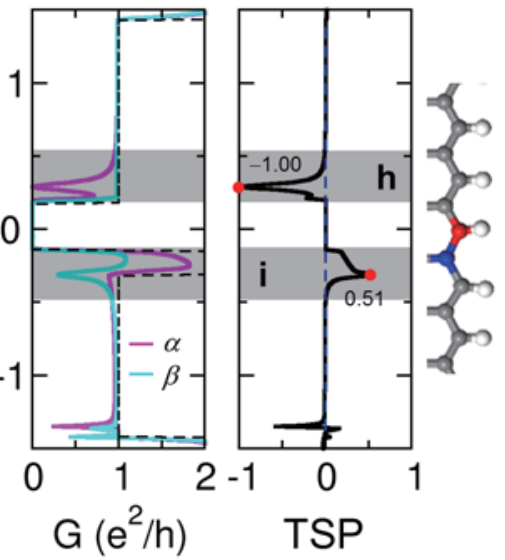

(h)

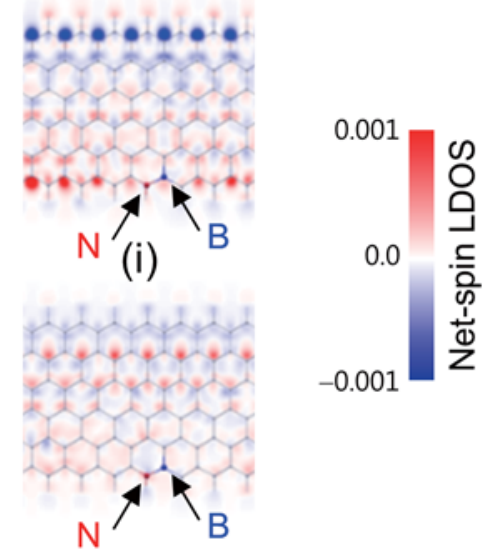

Fig. 3 Transmissions of (a) single B-doped, (b) single N-doped, and (c) B-N-complex doped 6-zGNRs for the AF spin configurations. Transmissions of pristine 6-zGNR are shown together (dotted lines). In (c), TSP values are also shown. Net-spin LDOS of (f) single B-doped $\left(E_{F}+0.5 \mathrm{eV} \sim E_{F}+0.85 \mathrm{eV}\right)$ and $(\mathrm{g})$ single N-doped $\left(E_{F}-0.85 \mathrm{eV} \sim E_{F}-0.5 \mathrm{eV}\right) 6-\mathrm{zGNRs}$ can be compared with those of $(\mathrm{d}, \mathrm{e})$ pristine and $(\mathrm{h}, \mathrm{i}) \mathrm{B}-\mathrm{N}$-complex-doped 6-zGNRs $\left(E_{F}+\right.$ $0.2 \mathrm{eV} \sim E_{F}+0.55 \mathrm{eV}$ for $(\mathrm{d}, \mathrm{h})$ and $E_{F}-0.45 \mathrm{eV} \sim E_{F}-0.15 \mathrm{eV}$ for $\left.(\mathrm{e}, \mathrm{i})\right)$. 
The above analysis of single $\mathrm{B} / \mathrm{N}$-doping cases suggests that localizing the doped-side edge defect states could be a route to generate more reliable spin-polarized currents. We now show that the B-N complex doping can achieve such a goal. The starting point of this argument is the B-N codoping-induced recovery of conductance (Fig. 3c) established above for aGNRs (see also Supplemental Data Fig. S4). We find that the conductance dips around $E_{F}+0.67 \mathrm{eV}$ (Fig. 3a) and $E_{F}-0.67 \mathrm{eV}$ (Fig. 3b) for the B-doped and N-doped 6-zGNRs, respectively, are restored back to the maximum quantum conductance of $1 G_{0}\left(=e^{2} / h\right)$. The crucial point is that the conductance recovery is slightly yet systematically incomplete for the spin channel spatially along the B-N doped GNR edge and energetically near the valence and conduction band-edge regions $\left(\sim E_{F}-0.19 \mathrm{eV}\right.$ and $\sim E_{F}+0.29 \mathrm{eV}$, respectively). Calculating the transport spin polarization (TSP) defined as

$$
\operatorname{TSP}(E)=\frac{T_{\alpha}(E)-T_{\beta}(E)}{T_{\alpha}(E)+T_{\beta}(E)}
$$

we particularly obtain TSP values of $-100 \%$ in the conduction band edge region. The origin of the sharp conductance dips appearing only for one spin channel in the valence and conduction band edges is indeed the spatially localized nature of B-N-complex defect states (spin currents along the undoped GNR edges are not affected), as can be visualized in the netspin LDOS plots (Figs. 3h and 3i). 
$\begin{array}{lll}\text { (a) B doped (FM) } & \text { (b) N doped (FM) } & \text { (c) B-N doped (FM) }\end{array}$

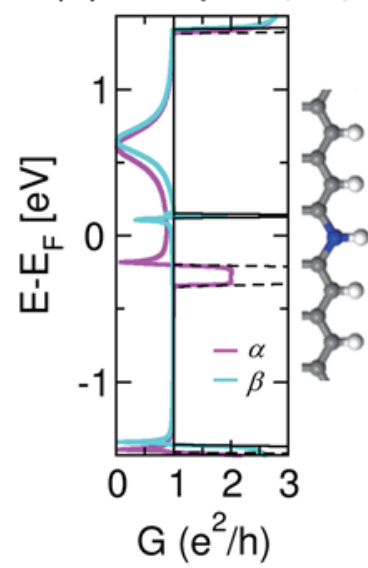

(d)

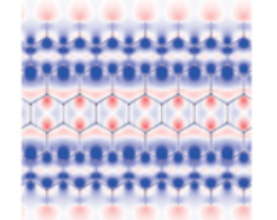

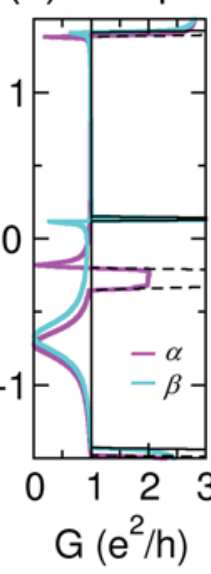

(e)

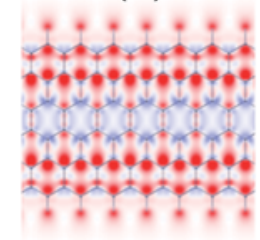

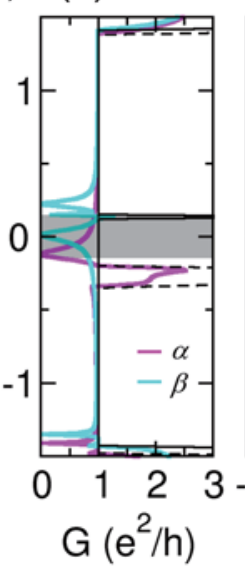

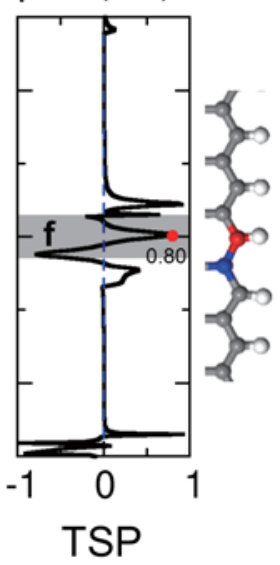

(f)

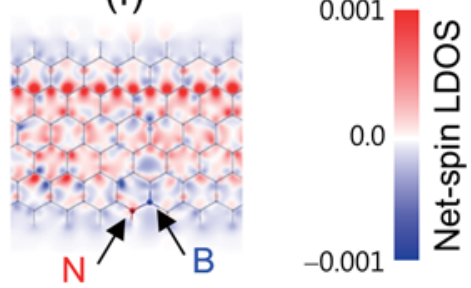

Fig. 4 Transmissions of (a) single B-doped, (b) single N-doped, and (c) B-N-complex-doped 6-zGNRs for FM spin configurations. Transmissions of pristine 6-zGNR are shown together (dotted lines). In (c), TSP values are also shown. Net-spin LDOS of (d,e) pristine $\left(E_{F}-0.45\right.$ $\mathrm{eV} \sim E_{F}$ and $E_{F} \sim E_{F}+0.45 \mathrm{eV}$, respectively) and (f) B-N-complex-doped ( $E_{F}-0.15 \mathrm{eV} \sim E_{F}$ $+0.15 \mathrm{eV}) 6-\mathrm{zGNRs}$.

In addition to the ground-state AF spin configuration, we have also considered the ferromagnetic (FM) spin configuration, which is energetically only slightly unstable [17] (Supplemental Data Table S1). Specifically, the energy difference was found to be only 0.002 eV per 12 graphene unit-cell 6-zGNR for the B-N-complex doped case. We find that, unlike the single B or single $\mathrm{N}$ doping counterparts (Figs. $4 \mathrm{a}$ and $4 \mathrm{~b}$, respectively), the B-N-complex doping can support $80 \%$ spin-polarized currents at $E_{F}$ (Fig. 4c) (see also Supplementary Data Fig. S5). As in the AF case, these spin-polarized currents are spatially as well as energetically localized (Fig. 4f), which makes them a source of spin-polarized electrons potentially useful 
in the nanoscale junction geometry.

(a) graphene / B-N zGNR (AF) / graphene
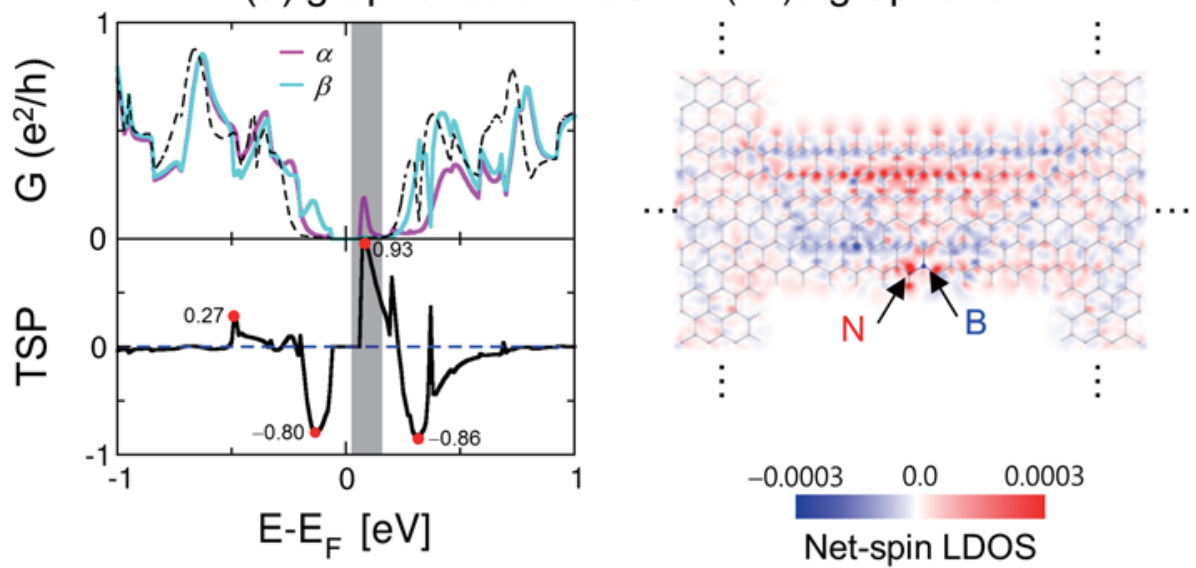

(b) B-N zGNR (FM) / BNNR / B-N zGNR (FM)
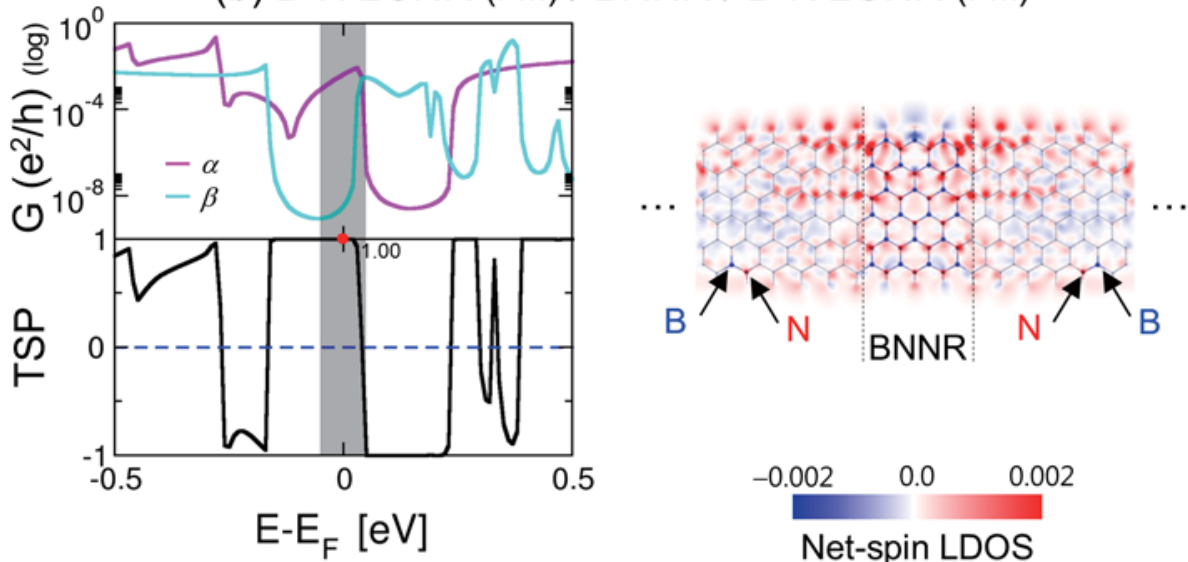

Fig. 5 (a) Spin-polarized transmissions and TSP obtained for the 11.5 unit-cell $(L=27.05 \AA)$

B-N-doped 6-zGNR (AF) channel sandwiched between graphene electrodes (left panel). Spin-unpolarized transmission from the junction based on a pristine 6-zGNR (AF) channel is shown for comparison (dotted line). Net-spin LDOS for the energy range $E_{F}+0.05 \mathrm{eV} \sim E_{F}+$ $0.25 \mathrm{eV}$ (right panel). (b) Spin-polarized transmissions and TSP obtained for the 3.5 unit-cell 6-BNNR channel $(L=18.70 \AA \AA)$ sandwiched between B-N-complex doped 6-zGNR (FM) electrodes (left panel). Net-spin LDOS for the energy range $E_{F}-0.05 \mathrm{eV} \sim E_{F}+0.05 \mathrm{eV}$ (right panel). 
We have confirmed the robustness of the above-described spin-polarized currents with respect to the variations in the GNR width and doping concentration (see Supplemental Data Figs. S6 and S7). Moreover, we also confirmed that the spin polarized currents survive in the heterojunction geometries, and here we describe two examples. In the first model (Fig. 5a), a B-N-complex edge-doped 6-zGNR in the AF spin configuration was sandwiched between two infinite graphene electrodes. Here, we found spin-polarized conductance with the TSP values of $+90 \%$ and $-80 \%$ in the conduction and valence band edges, respectively. In the second junction model (Fig. 5b), between two B-N-complex doped 6-zGNR electrodes in the FM spin configuration, we have inserted a hydrogen passivated boron nitride nanoribbon (BNNR) channel that has the same atomic configuration as 6-zGNRs and is an insulator with a wide bandgap of $\sim 4 \mathrm{eV}$. Again, we found that the spin-polarized currents injected from the B-N-complex doped zGNR electrodes tunnel through the BNNR channel with the TSP value of $+100 \%$ at $E_{F}$. For both cases, we can confirm in their LDOS that a fully spin-polarized channel appears in spite of the contacts with graphene electrodes or the BNNR channel.

\section{Conclusions}

In conclusion, based on first-principles calculations, we showed that the B-N-complex GNR edge doping is not only energetically favorable, but also can induce desirable and interesting quantum charge and spin transport properties. We predicted that it can generally recover the excellent charge transport capacity of pristine GNRs. In particular, for the zGNRs, the spatially localized nature of B-N defect states was shown to support robust and tunable spinpolarized currents. For the ground antiferromagnetic state, nearly $100 \%$ spin-polarized currents were predicted to flow along the B-N undoped edge near the conduction band-edge region. For the energetically comparable ferromagnetic spin configuration, fully spin- 
polarized currents along the B-N undoped edge were found to arise near $E_{F}$. It was confirmed that such spin-polarized states are robust with respect to the GNR width, doping concentration, and heterojunction geometries (both as an AF channel and FM electrodes). With the rapid progress in synthesis techniques [2, 14, 15, 19-21], GNRs have emerged as a promising ingredient of next-generation nanoelectronic and spintronic applications. The proposed B-N-complex edge doping may aid the fabrication of high-quality GNRs and benefit the design of graphene-based spintronic devices.

\section{Acknowledgements}

This research was supported by Global Frontier Program (No. 2013M3A6B1078881), Basic Science Research Grant (No. 2012R1A1A2044793), and Nano-Material Technology Development Program (No. 2012M3A7B4049888) of the National Research Foundation funded by the Ministry of Science, ICT and Future Planning of Korea. Computational resources were provided by the KISTI Supercomputing Center (No. KSC-2013-C3-046). 


\section{References}

[1] Terrones M, Botello-Méndez AR, Campos-Delgado J, López-Urías F, Vega-Cantú YI, Rodríguez-Macías FJ, et al. Graphene and graphite nanoribbons: Morphology, properties, synthesis, defects and applications. Nano Today. 2010;5(4):351-72.

[2] Li X, Wang X, Zhang L, Lee S, Dai H. Chemically Derived, Ultrasmooth Graphene Nanoribbon Semiconductors. Science. 2008;319:1229.

[3] Wang X, Ouyang Y, Li X, Wang H, Guo J, Dai H. Room-Temperature AllSemiconducting Sub-10-nm Graphene Nanoribbon Field-Effect Transistors. Phys Rev Lett. 2008;100:206803.

[4] Liang X, Wi S. Transport Characteristics of Multichannel Transistors Made from Densely Aligned Sub-10 nm Half-Pitch Graphene Nanoribbons. ACS Nano. 2012;6(11):9700-10.

[5] Son JG, Son M, Moon K-J, Lee BH, Myoung J-M, Strano MS, et al. Sub-10 nm Graphene Nanoribbon Array Field-Effect Transistors Fabricated by Block Copolymer Lithography. Adv Mater. 2013;25(34):4723-8.

[6] Ye Y, Gan L, Dai L, Meng H, Wei F, Dai Y, et al. Multicolor graphene nanoribbon/semiconductor nanowire heterojunction light-emitting diodes. J Mater Chem. 2011;21(32):11760-3.

[7] Son Y-W, Cohen ML, Louie SG. Half-metallic graphene nanoribbons. Nature. 2006;444(7117):347-9.

[8] Wimmer M, Adagideli İ, Berber S, Tománek D, Richter K. Spin Currents in Rough Graphene Nanoribbons: Universal Fluctuations and Spin Injection. Phys Rev Lett. 2008;100(17):177207.

[9] Kim WY, Kim KS. Prediction of very large values of magnetoresistance in a graphene nanoribbon device. Nat Nanotechnol. 2008;3(7):408-12. 
[10] Muñoz-Rojas F, Fernández-Rossier J, Palacios JJ. Giant Magnetoresistance in Ultrasmall Graphene Based Devices. Phys Rev Lett. 2009;102(13):136810.

[11] Rocha AR, Thiago BM, Fazzio A, Antônio JRdS. Disorder-based graphene spintronics. Nanotechnol. 2010;21(34):345202.

[12] Son Y-W, Cohen ML, Louie SG. Energy Gaps in Graphene Nanoribbons. Phys Rev Lett. 2006;97(21):216803.

[13] Barone V, Hod O, Scuseria GE. Electronic Structure and Stability of Semiconducting Graphene Nanoribbons. Nano Lett. 2006;6:2748.

[14] Han MY, Özyilmaz B, Zhang Y, Kim P. Energy Band-Gap Engineering of Graphene Nanoribbons. Phys Rev Lett. 2007;98(20):206805.

[15] Chen Y-C, de Oteyza DG, Pedramrazi Z, Chen C, Fischer FR, Crommie MF. Tuning the Band Gap of Graphene Nanoribbons Synthesized from Molecular Precursors. ACS Nano. 2013;7(7):6123-8.

[16] Fujita M, Wakabayashi K, Nakada K, Kusakabe K. Peculiar Localized State at Zigzag Graphite Edge. J Phys Soc Jpn. 1996;65(7):1920-3.

[17] Lee H, Son Y-W, Park N, Han S, Yu J. Magnetic ordering at the edges of graphitic fragments: Magnetic tail interactions between the edge-localized states. Phys Rev B. 2005;72(17):174431.

[18] Chae J, Jung S, Woo S, Baek H, Ha J, Song YJ, et al. Enhanced Carrier Transport along Edges of Graphene Devices. Nano Lett. 2012;12(4):1839-44.

[19] Baringhaus J, Ruan M, Edler F, Tejeda A, Sicot M, Taleb I, et al. Exceptional ballistic transport in epitaxial graphene nanoribbons. Nature. 2014;506(7488):349-54.

[20] Cai J, Ruffieux P, Jaafar R, Bieri M, Braun T, Blankenburg S, et al. Atomically precise bottom-up fabrication of graphene nanoribbons. Nature. 2010;466(7305):470-3. [21] Wang X, Ouyang Y, Jiao L, Wang H, Xie L, Wu J, et al. Graphene nanoribbons with 
smooth edges behave as quantum wires. Nat Nanotechnol. 2011;6(9):563-7.

[22] Wang X, Li X, Zhang L, Yoon Y, Weber PK, Wang H, et al. N-Doping of Graphene Through Electrothermal Reactions with Ammonia. Science. 2009;324(5928):768-71.

[23] Pantelides ST, Puzyrev Y, Tsetseris L, Wang B. Defects and doping and their role in functionalizing graphene. MRS Bull. 2012;37(12):1187-94.

[24] Yu S-S, Zheng W-T. Effect of N/B doping on the electronic and field emission properties for carbon nanotubes, carbon nanocones, and graphene nanoribbons. Nanoscale. 2010;2(7):1069-82.

[25] Martins TB, Miwa RH, da Silva AJR, Fazzio A. Electronic and Transport Properties of Boron-Doped Graphene Nanoribbons. Phys Rev Lett. 2007;98(19):196803.

[26] Huang B, Yan Q, Zhou G, Wu J, Gu B-L, Duan W, et al. Making a field effect transistor on a single graphene nanoribbon by selective doping. Appl Phys Lett. 2007;91(25):253122-3.

[27] Martins TB, da Silva AJR, Miwa RH, Fazzio A. $\sigma$ - and $\pi$-Defects at Graphene Nanoribbon Edges: Building Spin Filters. Nano Lett. 2008;8(8):2293-8.

[28] Yu SS, Zheng WT, Wen QB, Jiang Q. First principle calculations of the electronic properties of nitrogen-doped carbon nanoribbons with zigzag edges. Carbon. 2008;46:537.

[29] Biel B, Blase X, Triozon F, Roche S. Anomalous Doping Effects on Charge Transport in Graphene Nanoribbons. Phys Rev Lett. 2009;102:096803.

[30] Zheng XH, Rungger I, Zeng Z, Sanvito S. Effects induced by single and multiple dopants on the transport properties in zigzag-edged graphene nanoribbons. Phys Rev B. 2009;80(23):235426.

[31] Cruz-Silva E, Barnett ZM, Sumpter BG, Meunier V. Structural, magnetic, and transport properties of substitutionally doped graphene nanoribbons from first principles. Phys Rev B. 2011;83:155445. 
[32] Ceperley DM, Alder BJ. Ground State of the Electron Gas by a Stochastic Method. Phys Rev Lett. 1980;45(7):566-9.

[33] José MS, Emilio A, Julian DG, Alberto G, Javier J, Pablo O, et al. The SIESTA method for ab initio order- N materials simulation. J Phys Condens Matter. 2002;14:2745. [34] Troullier N, Martins JL. Efficient pseudopotentials for plane-wave calculations. Phys Rev B. 1991;43(3):1993-2006.

[35] Brandbyge M, Mozos J-L, Ordejón P, Taylor J, Stokbro K. Density-functional method for nonequilibrium electron transport. Phys Rev B. 2002;65(16):165401. [36] Kim Y-H, Tahir-Kheli J, Schultz PA, Goddard WA. First-principles approach to the charge-transport characteristics of monolayer molecular-electronics devices: Application to hexanedithiolate devices. Phys Rev B. 2006;73(23):235419.

[37] Kim Y-H, Jang SS, Jang YH, Goddard WA. First-Principles Study of the Switching Mechanism of [2]Catenane Molecular Electronic Devices. Phys Rev Lett. 2005;94(15):156801. 